\title{
双膦钉配合物 $\left[\mathrm{Ru}\left(\eta^{2}-\mathrm{CH}_{3} \mathrm{COO}\right)(\mathrm{dppm})_{2}\right]$ $\left[\mathrm{CH}_{3} \mathrm{COO}\right] \cdot 3 \mathrm{H}_{2} \mathrm{O}$ 的制备、晶体结构 和催化性能
}

高景星 王金枝 胡盛志 万惠霖

(厦门大学化学系,固体表面物理化学国家重点实验室, 厦门 361005)

\section{关键词双膦配体 钉配合物 氢化 晶体结构}

膦钉配合物在均相催化反应中的应用一直受到人们的注意. 早期合成的膦钉配合物 多半含有单齿的三苯基膦配体 ${ }^{[1]}$. 近年来, 由于多膦配体的应用, 使得许多低氧化态的金属 配合物得以稳定和被分离 ${ }^{[2.3]}$. 鉴于它们具有种种有趣的结构和在不对称合成中的重要应 用, 双膦与手性双膦金属配合物的研究十分活跃 ${ }^{[4]}$. 本文报道鳌合双膦钉配合物 $[\mathrm{Ru}$ $\left.\left(n^{2}-\mathrm{CH}_{3} \mathrm{COO}\right)(\mathrm{dppm})_{2}\right]\left[\mathrm{CH}_{3} \mathrm{COO}\right] \cdot 3 \mathrm{H}_{2} \mathrm{O}[\mathrm{dppm}=$ 双 - (二苯基膦 $)$ 甲烷 $]$ 的合成、晶体结构 和催化性能.

\section{1 题示物的合成、元素分析与谱学表征}

合成实验在氮气下进行. 溶剂甲苯经钠回流处理. 其余溶剂均为分析纯. $\mathrm{Ru}\left(\mathrm{CH}_{3} \mathrm{COO}\right)_{2}(\mathrm{P}$ $\left.\mathrm{h}_{3} \mathrm{P}\right)_{2}$ 按文献[1] 合成, dppm 为 Strem 公司产品. 元素分析数据由中国科学院上海有机化学研 究所提供. 分别用 Nicolet 740-FT 红外光谱仪和 JEOL 270 核磁共振仪测定 IR 光谱和 NMR 谱. $\mathrm{Ru}\left(\mathrm{CH}_{3} \mathrm{COO}\right)_{2}\left(\mathrm{Ph}_{3} \mathrm{P}\right)_{2}$ 和 $2 \mathrm{~mol} / \mathrm{L}$ 的 $\mathrm{dppm}$ 在甲苯中回流 $12 \sim 14 \mathrm{~h}$, 得黄色溶液. 减压除甲 苯, 分别用乙醚、正已烷洗后, 从二氯甲烷/ 正已烷重结晶得黄色晶体. 产率为 $78 \% \sim 83 \%$. $\mathrm{mp} 205 \sim 207{ }^{\circ} \mathrm{C}$. 元素分析 $\mathrm{C}_{54} \mathrm{H}_{50} \mathrm{O}_{4} \mathrm{P}_{4} \mathrm{Ru}$, 实验值 (计算值) \% C, 65.81(65.66); $\mathrm{H}, 5.22(5.07)$. IR (KBr): $3052 \mathrm{~m}, 2920 \mathrm{w}, 1584 \mathrm{~m}, 1568 \mathrm{~m}, 1522 \mathrm{~m}, 1484 \mathrm{~m}, 1454 \mathrm{~s}, 1436 \mathrm{vs}, 1392 \mathrm{~m}$, $1330 \mathrm{w}, 1190 \mathrm{w}, 1098 \mathrm{~s}, 998 \mathrm{w}, 732 \mathrm{~s}, 712 \mathrm{~s}, 692 \mathrm{vs}, 676 \mathrm{~m}, 510 \mathrm{vs}, 484 \mathrm{~cm}^{-1} .{ }^{1} \mathrm{H}$ NMR $\left(\mathrm{CDCl}_{3}\right): \eta^{2}-\mathrm{CH}_{3} \mathrm{COO}^{-}, \delta 1.77 \mathrm{~s}(3) ; \mathrm{CH}_{3} \mathrm{COO}^{-}, \delta 1.96 \mathrm{~s}(3) ;-\mathrm{CH}_{2}-, \delta 3.90 \mathrm{~m}(2), \delta 4.54 \mathrm{~m}$ (2); $\mathrm{C}_{6} \mathrm{H}_{5}{ }^{-}, \delta 6.10 \mathrm{~m}(4), 6.94 \sim 7.69 \mathrm{~m}(36) ;{ }^{31} \mathrm{P} \mathrm{NMR}\left(\mathrm{CDCl}_{3}\right): \delta-12.65 \mathrm{t}(2) ; J_{\mathrm{P}-\mathrm{P}}=39.1 \mathrm{~Hz}$; $\delta 6.87 \mathrm{t}(2), J_{\text {P.P }}=39.1 \mathrm{~Hz}$.

\section{2 晶体结构分析}

题示物 $\left(\mathrm{C}_{54} \mathrm{H}_{56} \mathrm{O}_{7} \mathrm{P}{ }_{4} \mathrm{Ru}, M_{r}=1042.01\right)$ 的单晶从二氯甲烷/ 正已烷重结晶获得. 选取一大小为

1994-12-22 收稿, 1995-07-18 收修改稿

*国家教育委员会留学回国人员基金资助项目 
$0.15 \mathrm{~m} \times 0.15 \mathrm{~m} \times 0.20 \mathrm{~mm}$ 的单晶安装在 CAD-4 四圆衍射仪 $(\mathrm{MoK} \alpha, \lambda=0.071073 \mathrm{~nm}$, 石墨单色 器) 上, 在 $\theta \leqslant 26^{\circ}$ 范围内收集到 10085 个独立衍射, 其中 $I \geqslant 3 \sigma(I)$ 的 4710 个衍射用于结构修 正. 强度数据经 $\mathrm{Lp}$ 因子和经验吸收校正. 晶体属单斜晶系, 空间群为 $P 2_{1} / n$. 唱胞参数: $a=1$, $2864(2), b=2.6695(5),{ }^{c} c=1.5356(4) \mathrm{nm}, \beta=107.30(2)^{\circ}, Z=4, D_{c}=1.375 \mathrm{~g} / \mathrm{cm}^{3}, F(000)=2160$, $\mu=4.786 \mathrm{~cm}^{-1}$. 在 80486 微机上运行 MoLEN 程序包, 从直接法解出全部非氢原子坐标, 再从 差分 Fourier 图求得部分氢原子坐标, 其余氢原子由加氢程序得到. 对非氢原子采用各向异 性热参数进行单位权重全矩阵最小二乘修正, 全部氢原子未参与修正而仅参加结构因子的计 算, 最后偏离因子 $R=0.062$, 而差值电子密度图上最高峰为 $1.03 \times 10^{3} \mathrm{e} / \mathrm{nm}^{3}$.

\section{3 氢化实验}

氢化实验在一容量为 $250 \mathrm{~mL}$ 的高压釜中进行. 温度精确至 $\pm 1^{\circ} \mathrm{C}$, 转速为 $500 \mathrm{r} / \mathrm{min}$. 按 常规的加压氢化实验进行操作, 产物经气相色谱定量.

\section{4 晶体结构描述与催化性能讨论}

晶体结构分析结果表明, 题示物具有离子型结构. 钉阳络离子 $\left[\mathrm{Ru}\left(\eta^{2}-\mathrm{CH}_{3} \mathrm{COO}\right)(\mathrm{dppm})_{2}\right]^{+}$ 的构型如图 1 所示, $\mathrm{Ru}$ 原子呈六配位而总体具有通过 $\mathrm{Ru}, \mathrm{Cl}$ 和 $\mathrm{C} 2$ 原子的膺二次轴对

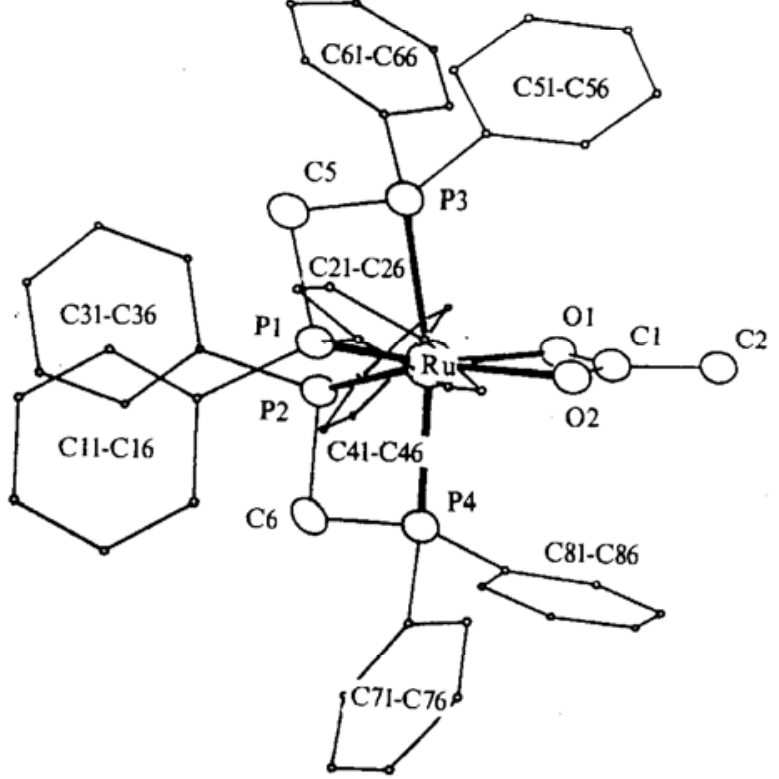

图 1 钉阳络离子 $\left[\mathrm{Ru}\left(\eta^{2}-\mathrm{CH}_{3} \mathrm{COO}\right)(\mathrm{dppm})_{2}\right]^{+}$的构型

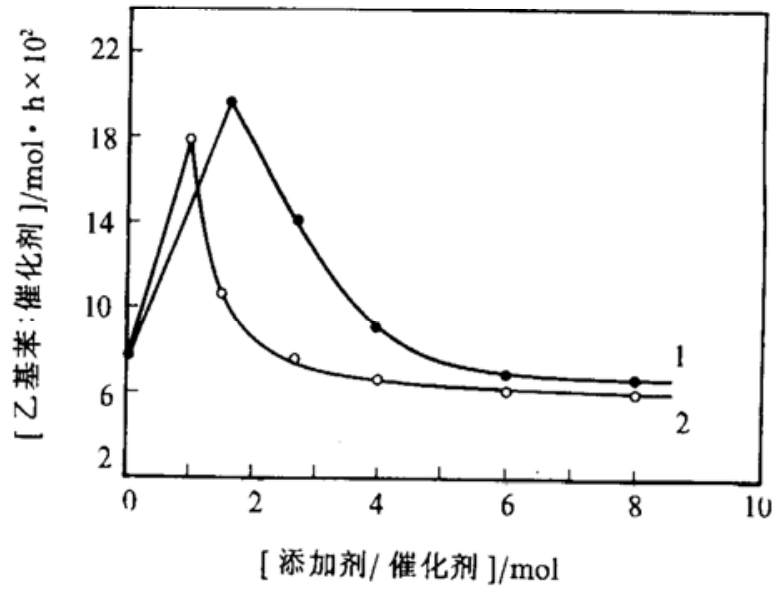

图 2 添加剂对氢化活性的影响 反应条件: 催化剂为 $\left[\mathrm{Ru}\left(\eta^{2}-\mathrm{CH}_{3} \mathrm{COO}\right)(\mathrm{dppm})_{2}\right]\left[\mathrm{CH}_{3} \mathrm{COO}\right]$, $0.0125 \mathrm{mmol}$, 苯乙烯为 $50 \mathrm{mmol}, 30^{\circ} \mathrm{C}$;

氢压为 $3.0 \mathrm{MPa}$; 时间为 $1 \mathrm{~h}$ 1 为添加剂为 $\mathrm{AgNO}_{3}, 2$ 为添加剂为 $\mathrm{NH}_{4} \mathrm{PF}_{6}$

称性. 表 1 为主要链长和键角值. C5 和 C6 与 P 原子形成的键角分别为 92.2(5) 和 92.7(5) ${ }^{\circ}$, 以保证 $\mathrm{P}$ 原子与 $\mathrm{Ru}$ 配位从而极大偏离正四面体角. 其余键长和键角均落在正常值范围. 除与 $\mathrm{Ru}$ 原子呈双齿配位的 $\mathrm{CH}_{3} \mathrm{COO}^{-}$外, 晶胞中还有未参与配位的 $\mathrm{CH}_{3} \mathrm{COO}^{-}$与水分子形成 $\mathrm{O}-\mathrm{H} \cdots \mathrm{O}$ 氢键体系.

在反应温度 $30 \sim 100{ }^{\circ} \mathrm{C}$, 氢压 $2.0 \sim 5.0 \mathrm{MPa}$ 的范围内, 考察了题示物催化 $\alpha, \beta$-不饱和 酸和几种醛、酮的选择氢化, 其结果如表 2 所示. 由表 2 看出, 题示物催化苯乙烯和丙烯酸的 
表 1 主要键长 $10 \mathrm{~nm}$ 和键角 $\left({ }^{\circ}\right)$

\begin{tabular}{|c|c|c|c|c|c|c|}
\hline $\mathrm{Ru}$ & P1 & $2.277(3)$ & $\mathrm{P} 1$ & $\mathrm{Ru}$ & P2 & $92.02(8)$ \\
\hline $\mathrm{Ru}$ & $\mathrm{P} 2$ & $2.277(3)$ & P1 & $\mathrm{Ru}$ & P3 & $70.2(1)$ \\
\hline $\mathrm{Ru}$ & P3 & $2.376(3)$ & P1 & $\mathrm{Ru}$ & P4 & $102.2(2)$ \\
\hline $\mathrm{Ru}$ & P4 & $2.346(4)$ & $\mathrm{Pl}$ & $\mathrm{Ru}$ & 01 & $160.8(2)$ \\
\hline $\mathrm{Ru}$ & 01 & $2.188(8)$ & PI & $\mathrm{Ru}$ & 02 & $107.2(2)$ \\
\hline $\mathrm{Ru}$ & 02 & $2.192(7)$ & P2 & $\mathrm{Ru}$ & P3 & $100.8(2)$ \\
\hline P1 & $\mathrm{C} 5$ & $1.89(2)$ & P2 & $\mathrm{Ru}$ & P4 & $70.5(1)$ \\
\hline $\mathrm{P} 1$ & $\mathrm{C} 11$ & $1.79(1)$ & P2 & $\mathrm{Ru}$ & 01 & $104.6(2)$ \\
\hline P1 & $\mathrm{C} 21$ & $1.82(1)$ & P2 & $\mathrm{Ru}$ & 02 & $157.0(2)$ \\
\hline P2 & C6 & $1.84(2)$ & P3 & $\mathrm{Ru}$ & P4 & $168.63(9)$ \\
\hline P2 & $\mathrm{C} 31$ & $1.84(1)$ & P3 & $\mathrm{Ru}$ & 01 & $96.8(3)$ \\
\hline $\mathrm{P} 2$ & C41 & $1.824(9)$ & P3 & $\mathrm{Ru}$ & 02 & $97.6(3)$ \\
\hline P3 & $\mathrm{C} 5$ & $1.83(1)$ & P4 & $\mathrm{Ru}$ & 01 & $92.7(3)$ \\
\hline P3 & C51 & $1.82(1)$ & P4 & $\mathrm{Ru}$ & 02 & $92.7(3)$ \\
\hline P3 & C61 & $1.82(1)$ & 01 & $\mathrm{Ru}$ & 02 & $59.4(2)$ \\
\hline P4 & C6 & $1.85(2)$ & $\mathrm{Ru}$ & P1 & C5 & $96.4(3)$ \\
\hline P4 & C71 & $1.81(2)$ & $\mathrm{Ru}$ & P1 & $\mathrm{C} 11$ & $128.3(3)$ \\
\hline P4 & $\mathrm{C} 81$ & $1.81(2)$ & $\mathrm{Ru}$ & $\mathrm{P} 1$ & $\mathrm{C} 21$ & $116.6(4)$ \\
\hline 01 & $\mathrm{Cl}$ & $1.26(1)$ & C5 & P1 & C11 & $105.5(6)$ \\
\hline 02 & $\mathrm{C} 1$ & $1.29(1)$ & C5 & P1 & $\mathrm{C} 21$ & $103.9(4)$ \\
\hline 03 & $\mathrm{C} 3$ & $1.28(2)$ & $\mathrm{C} 11$ & $\mathrm{P} 1$ & $\mathrm{C} 21$ & $102.9(5)$ \\
\hline 04 & $\mathrm{C} 3$ & $1.24(2)$ & $\mathrm{Ru}$ & $\mathrm{P} 2$ & C6 & $97.8(3)$ \\
\hline $\mathrm{Cl}$ & $\mathrm{C} 2$ & $1.49(2)$ & $\mathrm{Ru}$ & $\mathrm{P} 2$ & $\mathrm{C} 31$ & $130.1(3)$ \\
\hline \multirow[t]{24}{*}{$\mathrm{C} 3$} & $\mathrm{C} 4$ & $1.54(2)$ & $\mathrm{Ru}$ & P2 & $\mathrm{C} 4 \mathrm{I}$ & $114.3(4)$ \\
\hline & & & C6 & P2 & $\mathrm{C} 31$ & $105.7(5)$ \\
\hline & & & C6 & P2 & $\mathrm{C} 41$ & $104.9(5)$ \\
\hline & & & $\mathrm{C} 31$ & P2 & C41 & $101.4(5)$ \\
\hline & & & $\mathrm{Ru}$ & P3 & C5 & $95.1(3)$ \\
\hline & & & $\mathrm{Ru}$ & P3 & C51 & $117.8(4)$ \\
\hline & & & $\mathrm{Ru}$ & P3 & C61 & $126.2(3)$ \\
\hline & & & C5 & P3, & C51 & $106.4(5)$ \\
\hline & & & $\mathrm{C} 5$ & $\mathrm{P} 3$ & C61 & $105.7(6)$ \\
\hline & & & C51 & P3 & C61 & $103.3(5)$ \\
\hline & & & $\mathrm{Ru}$ & P4 & C6 & $95.1(3)$ \\
\hline & & & $\mathrm{Ru}$ & P4 & $\mathrm{C} 71$ & $125.5(3)$ \\
\hline & & & $\mathrm{Ru}$ & P4 & $\mathrm{C} 81$ & $115.9(3)$ \\
\hline & & & $\mathrm{C} 6$ & P4 & C71 & $107.1(6)$ \\
\hline & & & $\mathrm{C} 6$ & P4 & C81 & 107.1(5) \\
\hline & & & $\mathrm{C} 71$ & P4 & C81 & $104.2(5)$ \\
\hline & & & 01 & $\mathrm{Cl}$ & 02 & $117.6(9)$ \\
\hline & & & 01 & $\mathrm{Cl}$ & $\mathrm{C} 2$ & $122(1)$ \\
\hline & & & 02 & $\mathrm{C} 1$ & $\mathrm{C} 2$ & $120(1)$ \\
\hline & & & 03 & $\mathrm{C} 3$ & 04 & $121(2)$ \\
\hline & & & 03 & $\mathrm{C} 3$ & $\mathrm{C} 4$ & $117(2)$ \\
\hline & & & 04 & $\mathrm{C} 3$ & $\mathrm{C} 4$ & $121(2)$ \\
\hline & & & $\mathrm{P} 1$ & $\mathrm{C} 5$ & P3 & $92.2(5)$ \\
\hline & & & $\mathrm{P} 2$ & C6 & 04 & $92.7(5)$ \\
\hline
\end{tabular}


表 $2\left[\mathrm{Ru}\left(\eta^{2}-\mathrm{CH}_{3} \mathrm{COO}\right)(\mathrm{dppm})_{2}\right]\left[\mathrm{CH}_{3} \mathrm{COO}\right]$ 催化几种底物的氢化 ${ }^{\mathrm{a})}$

\begin{tabular}{|c|c|c|c|c|c|c|c|}
\hline 序号 & 底物 & 产物 & $\begin{array}{l}\text { 温度 } \\
/{ }^{\circ} \mathrm{C}\end{array}$ & $\begin{array}{l}\text { 氢床 } \\
/ \mathrm{MPa}\end{array}$ & $\begin{array}{c}\text { 时间 } \\
/ \mathrm{h}\end{array}$ & $\begin{array}{c}\text { 转化率 } \\
1 \%\end{array}$ & $\begin{array}{c}\text { 催化转换数 } \\
/ \mathrm{h}^{-1}\end{array}$ \\
\hline 1 & 丙烯酸 & 丙 酸 & 65 & 2.0 & 1.0 & 30.5 & 610 \\
\hline 2 & $\alpha$-甲基丙㛓酸 & $\alpha$-甲基妠酸 & 65 & 2.0 & 1.0 & 13.1 & 262 \\
\hline 3 & 苯乙烯 & 乙基苯 & 30 & 3.0 & 1.0 & 39.2 & 784 \\
\hline 4 & 策乙酮 & 苯乙醇 & 100 & 5.0 & 2.0 & 0 & 0 \\
\hline 5 & 环已裯 & 环已醇 & 100 & 5.0 & 2.0 & 7.1 & 71 \\
\hline 6 & 糠 醛 & 糠 醇 & 65 & 3.0 & 2.0 & 11.3 & 113 \\
\hline 7 & 苯甲醛 & 苯甲醇 & 100 & 5.0 & 2.0 & 28.9 & 289 \\
\hline 8 & 邻羟基苯甲醛 & 邻楼基苯甲醇 & 100 & 5.0 & 2.0 & 21.1 & 211 \\
\hline
\end{tabular}

a) 反应条件: 催化剂, $0.0125 \mathrm{mmol}$; 底物为 $25.0 \mathrm{mmol}$; 底物 / 催化剂 $=2000: 1$ (摩尔比率); 溶剂为甲醇, $35.0 \mathrm{~mL}$

氢化最有效, 但对环已酮的氢化活性较低, 而对苯乙酮的氢化无活性. 添加适量的促进剂对 题示物催化苯乙烯的加氢反应有明显的作用, 参见图 2. 当添加剂 $\mathrm{AgNO}_{3}$ 或 $\mathrm{NH}_{4} \mathrm{PF}_{6}$ 与题示物 的摩尔比分别为 $1: 1$ 和 $1: 1.5$ 时, 氢化活性最佳. 超过上述比值时活性下降. 产生这种现象 的可能原因是作为活性中心的 $\mathrm{Ru}$ 原子呈六配位的配位饱和构型. 添加 $\mathrm{Ag}^{+}$或 $\mathrm{NH}_{4}^{+}$有可能 促使双齿配体 $\mathrm{CH}_{3} \mathrm{COO}^{-}$的开环或脱离, 从而生成配位不饱和的催化活性品种 ${ }^{[n]}$, 而添加过量 的 $\mathrm{Ag}^{+}$或 $\mathrm{NH}_{4}^{+}$将导致催化剂的分解并被还原成黑色的 $\mathrm{Ru}$ 颗粒, 因而活性下降.

\section{参考 文献}

1 Mitchell W R, Spenœer R, Wilkinson G. Carboxylates triphenylphosphine complexes of ruthenium, cationic triphenylphosphine complexes derived from them and their behaviour as homogeneous hydrogenation catalysts for alkeues. J C S Dalton Trans, 1973, (8): $846 \sim 854$

2 Fsther B, Boyar P, Harding A et al. Complexes of platinum metals. Part 31. Reactions of binuclear ruthenium (II, III) and rhodium(II) carboxylates with chelating diphosphines: X-ray crystal structure of(acetato-o, o') bis [bis (diphenylphosphino)-methane-P, $\mathrm{P}^{\prime}$ ] ruthenium (II) tetraphenylborate. J C S Dalton Trans, 1986, (9): 177I 1778

3 Jia Guochen, Amold L R, Brian S H et al. Synthesis and Characterization of ruthenium acetate complexes containing triphosphines. Inorg Chem, 1992, 31(5): $900 \sim 904$

4 Noyori Ryoji, Takaya Hidemasa. BINAP: An efficient chiral element for asymmetric catalysis. Acc Chem, 1990, 23(10): $345 \sim 350$

5 Suarez T, Fontal B, Garcia D. Hydrogenation reactions with $\mathrm{Rucl}_{2}(\mathrm{~L}-\mathrm{L})_{2}(\mathrm{~L}-\mathrm{L}=$ bidentate phosphine). J Mol Catal, 1986, 34(2): $163 \sim 171$ 